\title{
From mice to humans: developments in cancer immunoediting
}

\author{
Michele W.L. Teng, ${ }^{1,2}$ Jerome Galon, ${ }^{3}$ Wolf-Herman Fridman, ${ }^{4}$ and Mark J. Smyth ${ }^{2,5}$ \\ 'Cancer Immunoregulation and Immunotherapy Laboratory, QIMR Berghofer Medical Research Institute, Herston, Queensland, Australia. ${ }^{2}$ School of Medicine, University of Queensland, Herston, Queensland, \\ Australia. ${ }^{3}$ INSERM UMRS1138, Laboratory of Integrative Cancer Immunology, Paris, France. Université Paris Descartes, Paris, France. Cordeliers Research Centre, Université Pierre et Marie Curie Paris 6, Paris, \\ France. ${ }^{4}$ Laboratory of Cancer, Immune Control and Escape, INSERM U1138, Cordeliers Research Centre, Paris, France. Université Pierre et Marie Curie, UMRS 1138, Paris, France. Université Paris Descartes, \\ UMRS 1138, Paris, France. ${ }^{I}$ mmmunology in Cancer and Infection Laboratory, QIMR Berghofer Medical Research Institute, Herston, Queensland, Australia.
}

\begin{abstract}
Cancer immunoediting explains the dual role by which the immune system can both suppress and/or promote tumor growth. Although cancer immunoediting was first demonstrated using mouse models of cancer, strong evidence that it occurs in human cancers is now accumulating. In particular, the importance of CD8+ $T$ cells in cancer immunoediting has been shown, and more broadly in those tumors with an adaptive immune resistance phenotype. This Review describes the characteristics of the adaptive immune resistance tumor microenvironment and discusses data obtained in mouse and human settings. The role of other immune cells and factors influencing the effector function of tumor-specific CD8 ${ }^{+} \mathrm{T}$ cells is covered. We also discuss the temporal occurrence of cancer immunoediting in metastases and whether it differs from immunoediting in the primary tumor of origin.
\end{abstract}

\section{Introduction}

The role of the immune system in recognizing and controlling tumor growth is well supported (1). Key evidence over the past decade from mouse models and human cancer patients has shown that the immune system recognizes transformed cells, and now it is generally accepted that avoiding immune detection and elimination is a hallmark of cancer (2). Conversely, the immune system can also promote tumor progression by supporting chronic inflammation, shaping tumor immunogenicity, and suppressing antitumor immunity. This dual role of the immune system in suppressing or promoting tumor growth is termed cancer immunoediting and consists of three phases: elimination, equilibrium, and escape (1).

In this Review, we provide a brief summary of the three phases of cancer immunoediting. We then describe the characteristics of an adaptive immune resistance tumor microenvironment that impacts survival outcome including its makeup (immune contexture), the distilled prognostic histological score (immunoscore), and the presence of tertiary lymphoid structures (TLSs). Mouse and human studies support the cancer immunoediting role of $\mathrm{CD} 8^{+} \mathrm{T}$ cells in this type of tumor microenvironment, as opposed to others, and we also discuss the role of other immune cells and factors in influencing the function of effector $\mathrm{CD}^{+} \mathrm{T}$ cells in this context. Finally, we discuss the temporal occurrence of cancer immunoediting in metastases and whether it differs from its primary tumor of origin.

\section{The three Es of cancer immunoediting: immunity shaping tumor immunogenicity} Elimination. In the elimination phase, innate and adaptive immunity work together to destroy developing tumors long before they

Conflict of interest: Mark Smyth has a research agreement with Bristol-Myers Squibb. Reference information: J Clin Invest. 2015;125(9):3338-3346. doi:10.1172/JCI80004. become clinically apparent. Although the elimination phase has not been directly visualized in vivo, studies have demonstrated that immunodeficient mice (deficient for effector molecules such as IFNs and perforin; recognition pathways like NKG2D; or cell types such as T and NK cells) displayed earlier onset or greater penetrance of carcinogen-induced and spontaneous cancers compared with that seen in WT mice (reviewed in refs. 1, 3-6).

Equilibrium. Rare tumor cell variants not destroyed in the elimination phase can proceed into the equilibrium phase, where their outgrowth is prevented by immunologic mechanisms. In a 2007 study (7), WT mice treated with low-dose methylcholanthrene (MCA) were demonstrated to harbor occult cancer cells that adaptive immunity (e.g., T cells and IFN- $\gamma$ ) kept in check. A subsequent study (8) demonstrated that immune-mediated tumor dormancy was dictated by a balance between two opposing cytokines, IL-12 and IL-23 (9), and could last for much of the lifespan of a mouse. The existence of the equilibrium phase was additionally supported by observations in mice with p53-mutant tumors (8) and by two other studies in which a Th1 environment dictated the eventual outcome of dormant tumors $(10,11)$.

Escape. When tumors circumvent immune recognition and/ or destruction, they progress from the equilibrium to the escape phase, where they become clinically apparent. Tumors escape due to changes in their response to immunoselection pressures and/or to increased tumor-induced immunosuppression or immune system deterioration. The mechanisms of tumor cell escape can be classified into three categories, as shown in Figure 1. Over the past two decades, these pathways have been the subjects of intense investigation, with the aim of developing new cancer immunotherapies (reviewed extensively in refs. 1, 3, 5).

Regarding tumor editing in each of these three phases, tumors derived from immunodeficient mice were found to be more immunogenic than were similar tumors derived from immunocompetent 
A

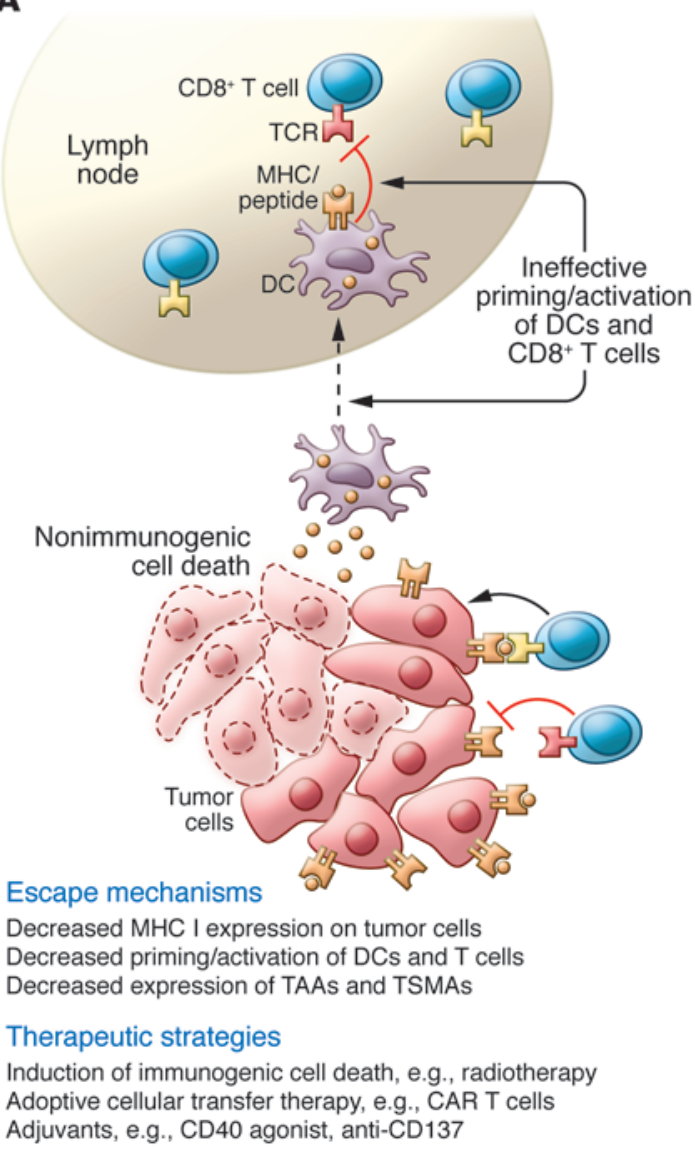

B

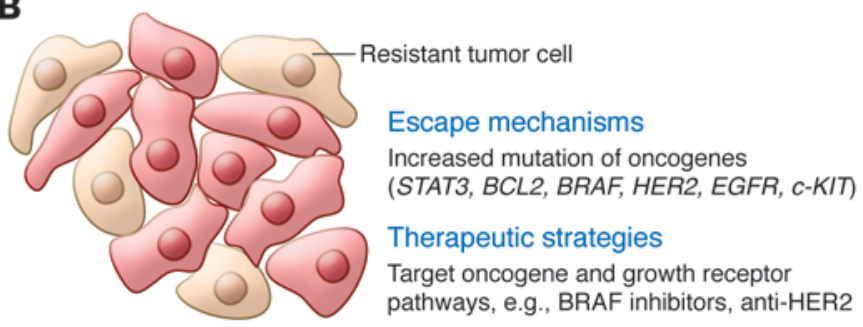

C

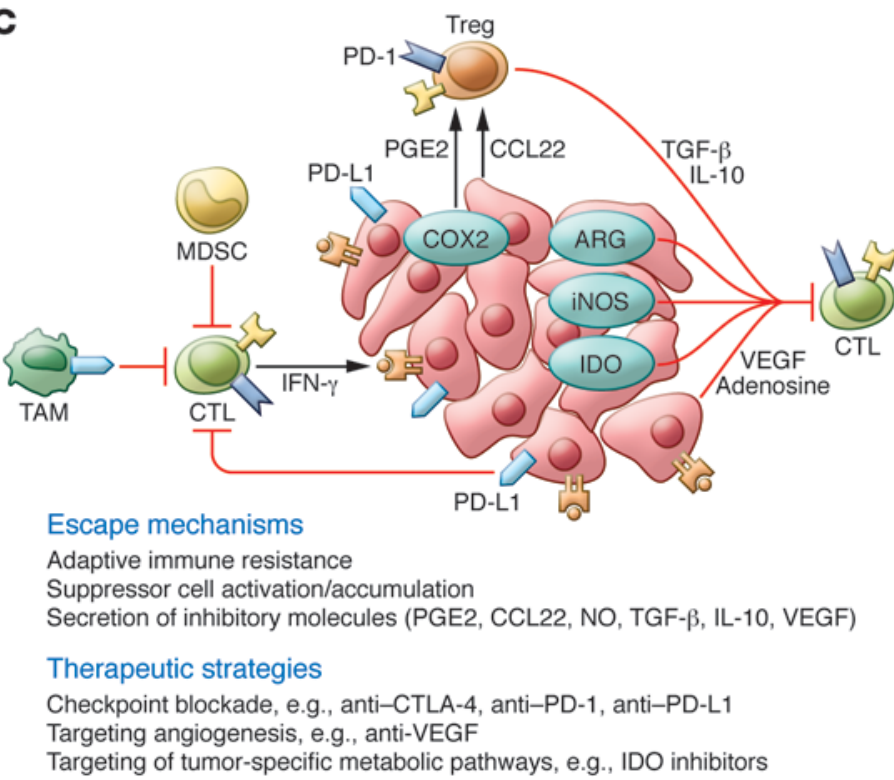

Figure 1. Major mechanisms of tumor escape and therapeutic options. The mechanisms of tumor cell escape can be classified into three major categories: (A) reduced immune recognition and immune cell stimulation through downregulation or loss of strong tumor antigens and antigen-presenting machinery or lack of costimulatory molecules; (B) upregulation of resistance mechanisms against the cytotoxic effectors of immunity (e.g., STAT3) or increased expression of prosurvival or growth factor genes (e.g., Bcl-2, Her2/neu); and (C) establishment of an immunosuppressive tumor microenvironment via (a) production of cytokines (e.g., VEGF, TGF- $\beta$ ) and metabolic factors (e.g., adenosine, PGE2); (b) induction and/or recruitment of Tregs and MDSCs; or (c) induction of adaptive immune resistance through ligation of inhibitory receptors (e.g., CTLA-4, PD-1, Tim-3) on immune effector cells. Over the past two decades, strategies to target these pathways have been the subject of intense investigation, and some of these are listed in the figure. iNOS, inducible NOS.

mice (1). Although T cells were inferred to sculpt tumor immunogenicity, it was unclear whether antigens expressed by emerging nascent tumor cells were recognized by T cells and could then be subsequently modulated in response to selection pressure. A study using an exome-sequencing approach demonstrated that sarcoma cells derived from MCA-inoculated mice expressed immunodominant neoantigens that could be immunoedited by $\mathrm{CD} 8^{+} \mathrm{T}$ cells, leading to tumor escape (12). Thus, the outgrowth of tumor cells lacking these strong antigens represents one mechanism of cancer immunoediting.

\section{Cancer immunoediting: from mice to humans}

The process of cancer immunoediting is obviously more difficult to assess in humans, given that they are an outbred population living in an uncontrolled environment; therefore, there are few examples of viable, specific immune gene loss (5). Nevertheless, the earliest clinical data supporting the immunoediting process in humans, including data from patients with severe immunodeficiencies, receiving organ transplants, spontaneously rejecting their tumors, or producing tumor-associated, antigen-specific antibodies, have been exten- sively reviewed and summarized (5). More specifically, early proof for the elimination phase of cancer immunoediting in humans also comes with the observation that clonal $\mathrm{T}$ cells were expanded in patients with spontaneously regressing melanoma lesions (13-15). Moreover, the presence of tumor-infiltrating $\mathrm{CD} 8^{+} \mathrm{T}$ cells and a Th1 immune signature is prognostic of improved disease-free survival (DFS) and overall survival (OS) compared with the classical tumor-node-metastasis (TMN) staging $(5,16)$. This information is captured at the stage of tumor escape, but predicts the ability of the natural immune reaction or therapies to drive the human tumor into an equilibrium or, indeed, eliminate the tumor altogether. Critically, the recent success of immune checkpoint inhibitors (e.g., antiCTLA-4 and anti-PD-1/-PD-L1) in the clinic (17) further demonstrates that cancer immunoediting occurs in patients with advanced cancers and that this process can be reset by therapy.

Immune contexture, immunoscore, and TLS: influencing survival outcome

A central feature of cancer immunoediting is that tumors express antigens (tumor-associated antigens [TAAs] or tumor-specific 
A

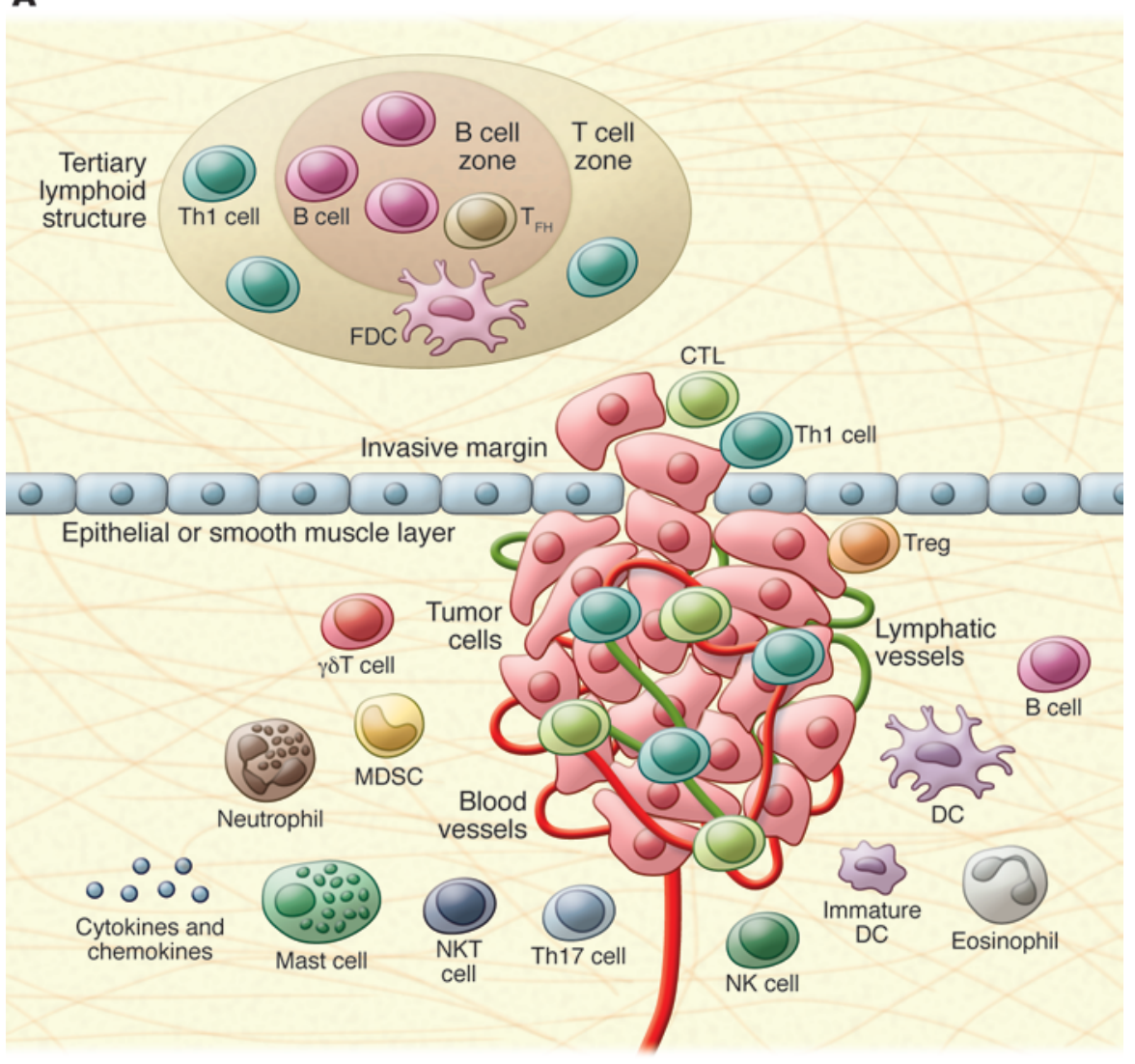

Figure 2. The immune contexture of the tumor microenvironment as a prognostic marker for long-term survival. (A) Cellular components of the tumor stroma include blood and lymphatic vessels, infiltrating and resident leukocytes, various populations of fibroblasts, and mesenchymal support cells unique to each tissue environment. The immune contexture is defined as the type, density, functional orientation, and location of immune cells within distinct tumor regions. A spectrum of soluble cytokines and chemokines regulate the entry of immune cells into tumors, which then have different effects on tumor progression. All types of immune cells are present in the tumor, including macrophages, DCs, mast cells, NK cells, naive and memory lymphocytes, $B$ cells, and effector $T$ cells (including various subsets of T cells: Th cells, Th1, Th2, Th17, Tregs, Tfh, and (TLs). Immune cells can be located in the core of the tumor, in the invasive margin, or in the adjacent TLSs. Few $C D 8^{+} T$ cells are seen in human TLSs, which are similar to secondary follicles in lymph nodes. TLSs contain naive and memory T cells, Tfh cells, B cells, and mature DCs. FDC, follicular DC. (B) High expression levels of various immune parameters that define a Th1 immune contexture, as well as the presence of Tfh cells, B cells, CXCL13, IL-21, and IL-15, are associated with prolonged DFS and/or improved OS in CRC (figures adapted from refs. 16, 23).
B

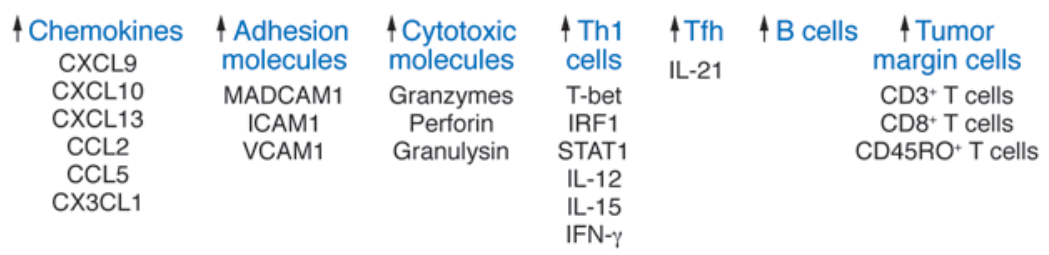

mutant antigens [TSMAs]) that can be recognized by $\mathrm{T}$ cells, resulting in their eventual destruction and/or selection of tumor escape clones. As such, the importance of $\mathrm{T}$ cells, in particular $\mathrm{CD}^{+} \mathrm{T}$ cells, in cancer immunoediting has been demonstrated in many preclinical mouse models of cancer (5). In humans, large annotated data sets have since demonstrated the presence of infiltrating $\mathrm{CD}^{+} \mathrm{T}$ cells in tumors. In this section of the Review, we discuss immune contexture, immunoscore, and the presence of tumor-adjacent TLSs that can predict patient survival and responses to immunotherapy (16).

Immune contexture. The immune contexture of human cancers defines a complex immunologic tumor microenvironment in which the location, density, and functional orientation of infiltrating hematopoietic cells correlate with the clinical outcome of patients (reviewed extensively in ref. 16). In most cancers, a strong infiltration of memory $\mathrm{CD}^{+} \mathrm{T}$ cells and a Th1 orientation correlated with a favorable prognosis in terms of progression-free survival (PFS) and/or OS (ref. 16 and Figure 2). Other parameters such as immune cells, cytokines, chemokines, and other factors also influence the generation of a clinically efficient immune microenvironment (Figure 2). In tumors, all types of immune cells can be found in various proportions and locations. They include myeloid cells, B cells, and all subsets of T cells (refs. 16, 18 , and discussed in further detail below).

Immunoscore. The "immunoscore" is a scoring system derived from the immune contexture $(16,19,20)$ and is a clinically useful prognostic marker (21) based on the enumeration of two lymphocyte populations (CD3 and CD8), in both the core of the tumor (CT) and in the invasive margin (IM) of tumors. The immunoscore provides a score ranging from immunoscore 0 , in which low densities of both cell types are found in both regions, to immunoscore 4 , in which high densities are found in both regions. Immunoscore classification was shown to have a prognostic significance in all stage I, II and III patients with colorectal cancer (CRC) that was superior to that of the American Joint Committee on Cancer/ Union for International Cancer Control (AJCC/UICC) TNM (T stage, $\mathrm{N}$ stage, and tumor differentiation) classification system $(22,23)$. Tumor invasion was shown to be statistically dependent on the host's immune reaction. It is believed that the ability of effector memory $\mathrm{T}$ cells to recall previously encountered antigens 
and traffic into tumors may lead to long-term immunity in human cancer $(24,25)$. Now, a clinical validation of the immunoscore with standardized procedures is necessary to reach clinical applicability for individual patients with CRC and other cancers (26, 27). The immunoscore also provides a tool and targets for novel therapeutic approaches, including immunotherapy (as recently illustrated in clinical trials boosting $\mathrm{T}$ cell responses with antiCTLA-4, anti-PD-1, and anti-PD-L1) (28-31).

Tumor-adjacent TLSs. In situations of chronic stimulation of the immune system, such as in autoimmune and inflammatory diseases or in transplanted organs, organized lymphoid structures can form in these pathological locations, displaying all characteristics of the immunity-generating sites of secondary lymphoid organs (reviewed in ref. 32). These TLSs can represent a very powerful means to generate effective, local immune reactions. In fact, they precede the appearance of lymph nodes in evolution (33), and lung viral infections can be eradicated by local TLSs in lymph node-deficient mice (34). TLSs are consistently found in the tumor microenvironment of most human cancers (32).

TLSs are composed of a T and a B cell zone, where $\mathrm{T}$ cells are in contact with mature DCs, whereas B cells interact with follicular DCs. These TLSs are surrounded by peripheral lymph node addressin-expressing high endothelial venules (HEVs), which are likely sites of entry of naive lymphocytes from the blood. All lymphocytes in TLSs express the peripheral lymph node addressin (PNAd) receptor CD62-L. Naive T cells are likely instructed by contacting DCs presenting TAAs and central memory and effector memory $\mathrm{T}$ cells, all of which are found in TLSs. In tumors, B cells are almost exclusively found in TLS germinal centers, where they express the somatic hypermutation and switch recombination enzyme activation-induced cytidine deaminase (AICDA). Plasma cells are seen at the periphery of the TLS and have been reported to produce antitumor-specific antigens in non-smallcell lung cancer (NSCLC) (35). One can therefore hypothesize that efficient antitumor reactions can be generated within TLSs, where DCs instruct lymphocytes, which, having entered directly through HEVs, escape the suppressive milieu of the tumor microenvironment (32).

TLSs appear to play a role in the anticancer immune response. Indeed, in melanoma, breast, lung, and colorectal carcinomas, high densities of TLSs have been reported to correlate with a favorable prognosis (36). Strikingly, TLSs are induced in cervical carcinomas (37) or pancreatic ductal cancer (38) upon vaccination with TSMAs. That TLSs are necessary for generating clinically efficient immune reactions is supported by the fact that a high density of $\mathrm{CD}^{+} \mathrm{T}$ cells is associated with longer patient survival when tumors display high TLS densities in lung (39), colorectal (40), and renal cell cancers (41). By contrast, they correlate with shorter survival in lung (39) and clear cell renal cell carcinoma (41) with low numbers of intratumoral TLSs. Recently, the presence of TLSs has been reported in some breast cancers, which suggests the presence of organized immunity and the generation of memory B cells within the tumor (42). Therefore, identification, enumeration, and structural analyses of TLSs are useful tools to predict patient prognosis and immunotherapy follow-up, but also to identify tumor-specific clones of T and B cells.

\section{Tumors displaying an adaptive immune resistance microenvironment}

Although a strong infiltration of $\mathrm{CD}^{+} \mathrm{T}$ cells and a Th1 orientation correlated with favorable prognosis in terms of DFS and OS, their effector function in tumors can be blunted through various immunosuppressive strategies mediated by tumors (43). One mechanism, termed adaptive immune resistance, represents the attempt of cancer cells to evade the immune system $(44,45)$. During this process, recognition of tumor cells by activated $\mathrm{T}$ cells results in the production of IFN- $\gamma$. This upregulates PD-L1 (B7-H1) on tumor cells and tumor-infiltrating immune cells and binds it to PD-1, an inhibitory immune checkpoint receptor expressed on activated T cells to attenuate their effector function. These receptors are normally used for maintaining self-tolerance and modulating the duration and amplitude of the physiological immune response but have been hijacked by tumors as a means of avoiding immune destruction. Indeed, high levels of PD-L1 on tumor-associated myeloid cells and draining lymph node DCs (46) and B7-H4 (another B7 family member) on macrophages (47) are important immune escape mechanisms in human cancers. It is now clear that engagement of checkpoint receptors on activated $\mathrm{CD}^{+} \mathrm{T}$ cells represents a major mechanism of tumor-induced immunosuppression. Indeed, over the past five years, immune checkpoint inhibitors anti-CTLA-4 (ipilimumab), anti-PD-1 (pembrolizumab, nivolumab), and anti-PD-L1 (MPDL3280A) have had remarkable success in inducing durable clinical responses across a broad spectrum of malignancies $(29,31$, 48-50) and have revolutionized the field of cancer immunology. PD-1/PD-L1 blockade is thought to operate predominantly in the tumor microenvironment, since PD-L1 ligand is commonly overexpressed by tumor cells and myeloid cells within human tumors (50); in comparison, CTLA-4 and its ligands are not as tumor localized.

The respective roles of malignant cells and the host in shaping the tumor/immune contexture is beginning to be uncovered by analysis of cancer subgroups based on immune microenvironments that are defined by PD-L1 expression and the presence of tumor-infiltrating lymphocytes (TILs). On the basis of the observation that patients' responses to anti-PD-1 were significantly associated with PD-1 expression on TILs and PD-L1 expression on tumor cells $(44,51,52)$, Taube et al. proposed stratifying the tumor microenvironment into four types. Type 1 is PD-L1 positive, with TILs driving adaptive immune resistance; type II is PD-L1 negative, with no TILs, indicating immune ignorance; type III is PD-L1 positive, with no TILs, indicating intrinsic induction; and type IV is PD-L1 negative, with TILs, indicating the role of other suppressor(s) in promoting immune tolerance. PD-L1 can also be expressed constitutively on cancer cells through poorly characterized oncogenic signaling pathways $(53,54)$, and type I and III tumor microenvironments may include such tumor cells. For the rest of this Review, we will discuss the role of cancer immunoediting in tumors with the type I adaptive immune resistance phenotype, given that evidence for cancer immunoediting in this subgroup is strongest.

\section{Cancer immunoediting in tumors with an adaptive immune resistance phenotype}

Since Taube's initial stratification, clinical studies have gone on to further define the immune profile of patients who respond to therapies targeting the $\mathrm{PD}-1 / \mathrm{PD}-\mathrm{L} 1$ pathway. While tumor 
expression of PD-L1 was thought to be an indicator of response, Herbst et al. demonstrated that PD-L1 expression on immune cells (particularly myeloid) in different cancers (NSLC, renal cell carcinoma [RCC], melanoma, head and neck squamous cell carcinoma [HNSCC], gastric cancer, CRC, pancreatic cancer) was also a key predictor of clinical activity $(50,52)$, adding further complexity (45). Tumeh et al. also determined that the pretreatment number of IM PD-1-expressing CD $8^{+} \mathrm{T}$ cells correlated with clinical responses in metastatic melanoma patients treated with anti-PD-1 (pembrolizumab) (52). This suggested the presence of a specific immune response to tumor antigens. Using next-generation sequencing, they demonstrated that pretreated CD8 ${ }^{+} \mathrm{T}$ cells had a more restricted $\mathrm{T}$ cell receptor $\beta$ (TCR $\beta$ ) chain usage, indicating that clonality correlated with clinical response to anti-PD-1 (52). This validates a previous study showing that PD-1 identified patient-specific $\mathrm{CD}^{+}$tumor-reactive $\mathrm{T}$ cells infiltrating human melanoma (55). Similar observations were also made in a subgroup of CRC patients who displayed microsatellite instability (MSI) characterized by a high number of neoantigens as a consequence of a DNA repair defect (56). This subgroup displayed the adaptive immune resistance phenotype characterized by a high infiltration of $\mathrm{T}$ cells, particularly $\mathrm{CD} 8^{+} \mathrm{T}$ cells, and these patients had a good prognosis with few metastases $(57,58)$. This observation was recently confirmed by Llosa et al., who reported that almost all CRCs with MSI (CRC-MSI), but not microsatellite stable (MSS) CRC (CRC-MSS), displayed high infiltration with activated CD8 ${ }^{+}$ cytotoxic $\mathrm{T}$ lymphocytes (CTLs) and activated Th1 cells characterized by IFN- $\gamma$ production and the Th1 transcription factor T-bet (57). Interestingly, the upregulation of multiple immune checkpoints (PD-1, Tim-3, LAG-3, CTLA-4, IDO) was only found in CRC-MSI patients. This may explain why MSI tumors are not naturally eliminated, despite an immune-promoting Th1/CTL microenvironment. Given this compelling evidence, two clinical trials (ClinicalTrials.gov identifiers NCT01876511 and NCT02060188) have commenced in order to test the efficacy of anti-PD-1 in CRC patients stratified by their MSI status (57).

Neoantigen discovery. While a number of TAAs have been identified since the 1990s (e.g., NY-ESO-1, MAGE), TSMAs have been harder to discover due to the labor-intensive process required to identify them. However, with advances in genomics and bioinformatics technology, a number of recent studies in mice and humans have identified TSMAs $(59,60)$. These studies further strengthen the idea that "passenger" mutations as opposed to "driver" mutations (which contribute directly to cancer initiation and progression) can function as neoantigens that induce tumor immunity before or after therapeutics such as ipilimumab. Using the same MCA-induced tumor model that Matsushita et al. used to study immunoediting of immunodominant neoantigens by $\mathrm{CD}^{+} \mathrm{T}$ cells (12), Gubin et al. showed that even escape tumors harbor TSMA-recognizing $\mathrm{T}$ cells, but are inhibited by immune checkpoints (PD-1 and CTLA-4) (59). Such TSMAs can act as therapeutic vaccines, as evidenced by their ability to cause established tumor rejection with an efficacy similar to that of immune checkpoint blockade therapy in tumor-bearing mice. Given the heterogeneity of tumors (61), it would be wise to vaccinate with a number of TSMAs, if possible, to reduce the emergence of escape variants. This is illustrated in a case report of a patient with melanoma who expressed a number of TAAs (NY-ESO-1, MAGE-C1, and Melan-A) prior to immunization with an NY-ESO-1 vaccine (62). In another case report, analyses of three consecutive lesions obtained within one year of a patient's developing stage IV melanoma revealed a gradual loss of immunogenicity that culminated in complete $\mathrm{T}$ cell resistance of the tumor cells caused by an irreversible HLA class Inegative phenotype (63). Overall, these studies provide evidence that tumors can express antigens recognizable by $\mathrm{CD}^{+} \mathrm{T}$ cells and that they are critical in the immunoediting of tumors with an adaptive immune resistance phenotype.

Does cancer immunoediting occur in epithelial cancers? Many of the principles of cancer immunoediting were proposed and demonstrated in mice using the chemically induced MCA sarcoma model $(7,64)$. These sarcomas are considered immunogenic and have a high mutational load similar to that of ultraviolet- and other carcinogen-induced human cancers (12). As such, a common criticism for the cancer immunoediting hypothesis is a lack of evidence that it holds true for epithelial cancers caused by simple drivers, which often have a lower mutational load (65). This may significantly limit the number of neoantigens capable of being presented on the tumor's HLA class I alleles.

There is now increasing evidence to support the occurrence of cancer immunoediting in epithelial cancers. Recently, genetic findings provided evidence for immunoediting in tumors and uncovered mechanisms of tumor-intrinsic resistance to cytolytic activity (66). In a patient with metastatic cholangiocarcinoma, a cancer with a low mutational load, researchers detected the presence of a specific $\mathrm{CD} 4^{+} \mathrm{T}$ cell recognizing a mutation in the ERBB2-interacting protein (ERBB2IP) (67). Similarly, high levels of CD8 ${ }^{+} \mathrm{PD}-1^{+} \mathrm{T}$ cells have been observed in small numbers of patients with CRC-MSS, who generally have a lower mutational load, suggesting that good $\mathrm{T}$ cell neo-epitopes can be generated if the mutations are appropriately positioned (57). Epithelial ovarian cancer (EOC), classically thought to be a nonimmunogenic cancer, provides further examples of cancer immunoediting (68), including tumor-specific CD8 ${ }^{+}$ $\mathrm{T}$ cells that recognize mutated oncogenes such as HER2 (69). An increasing number of large breast cancer data sets have reported the correlation of $\mathrm{T}$ cell infiltrates with better clinical outcomes, including in HER2-positive breast cancer patients, for whom an association between immunity and better survival after treatment with chemotherapy and anti-HER2 therapies has been reported (42). High levels of $\mathrm{T}$ cell checkpoint receptors have also been detected in HER2-overexpressing breast cancer at the mRNA level as well as in triple-negative breast cancer (TNBC) (70). Anti-HER2 and anti-PD-1 have been shown to be synergistic in mouse models (71), and recently, anti-PD-1 (pembrolizumab) demonstrated clinical activity in TNBC (72). This potentially opens up new treatment avenues for patients with breast cancer.

\section{Other parameters impacting CD8 ${ }^{+} \mathrm{T}$ cells in cancer immunoediting}

We now discuss how the presence of immune cells and other parameters modulate the positive clinical impact of memory CD8 T cells and affect their efficacy as potential antitumor effector cells.

Myeloid cells. In general, high densities of myeloid cells, i.e., macrophages and myeloid-derived suppressor cells (MDSCs), correlate with poor prognosis (73). When it has been characterized, it appears 
that the negatively impacting macrophages are of the M2 phenotype (74). In any case, the correlation between macrophage density and patient survival is less significant than that of $\mathrm{T}$ cells, particularly $\mathrm{CD}^{+} \mathrm{T}$ cells (18), and it has been proposed that clinical outcome could be better predicted by the ratio of the densities of $\mathrm{CD}^{+}$ $\mathrm{T}$ cells/macrophages $(75,76)$. In contrast, a high density of mature DCs is generally associated with longer survival, as in NSCLC (77), breast cancer (78), or melanoma (78), although the density of myeloid cells expressing the DC-lysosomal-associated membrane protein (LAMP) marker was found to correlate with shorter survival in RCC $(41,79)$. In a mouse model of ovarian cancer, it was shown that phenotypically divergent DCs drive both immunosurveillance and accelerated tumor growth (80). The switch from one phenotype to the other coincided with an increasingly immunosuppressed tumor microenvironment. In addition to macrophages and DCs, emerging data have revealed a role of neutrophil extracellular traps (NETs) in cancer immunoediting. These are neutrophil-derived structures composed of extruded DNA, decorated with antimicrobial proteins. Although they are generally formed in response to infectious stimuli, they have been reported to sequester circulating tumor cells and promote metastases $(81,82)$.

$C D 4^{+} T$ cell subsets. Compared with myeloid cells, the effects of $\mathrm{T}$ cell subsets in the tumor/immune microenvironment appear much less straightforward (16). The Th2 signature correlated with favorable prognosis in Hodgkin lymphoma (83) and breast cancer (84), but shorter survival in ovarian (85), pancreatic (86), and gastric cancers (87). Conversely, a Th17 signature was found to be deleterious in colorectal (88), lung (89), and hepatocellular carcinoma (90) and beneficial in ovarian (91), esophageal (92), and gastric (93) cancers. These findings highlight the fact that the cancer type may influence the clinical impact of the tumor/immune microenvironment on the apparent balance between Th2 and Th17 cells. Thus, when a Th2 signature was associated with poor prognosis, such as in ovarian, pancreatic, and gastric cancers, it was reported by independent groups that, in these cancer types, a Th17 signature correlated with a favorable clinical outcome. These observations suggest that the clinical impact of immune cell populations is highly dependent on the overall immune microenvironment, which is likely orchestrated by the cancer cell type (tissue of origin or organ) and by the genetics of the tumor. Intratumoral Tregs are even more complex, since they have been reported to be associated with poor or favorable prognosis, not only in different cancer types, but also in the same cancer $(94,95)$. It may reflect the heterogeneity of Treg subsets (96) in the tumor microenvironment or the selectivity of Treg markers (97).

Other immune cells. A number of other types of lymphocytes also contribute to the immune contexture of cancer, cancer immunoediting, and patient outcome. Unlike what has been observed in mouse models (75), high densities of B cells and T follicular helper (Tfh) cells correlated with longer survival in NSCLC (35), breast cancers (98), and CRC (18). In contrast, the impact of NK cell density is either weakly favorable (79) or null (99), depending on the balance of the expression of activating and inhibitory receptors that generally regulate the effector function of NK cells $(99,100)$. The mouse lectin-like Ly 49 family receptors, which are similar to human killer inhibitory receptors in their capacity to bind MHC class I molecules, largely control the self-reactivity of NK cells.
The importance of Ly49 in NK cell-mediated tumor immunosurveillance and MHC I-directed tumor editing was recently demonstrated in a study using Ly49-deficient mice (101). Another study demonstrated that human tumors reduced activating NK cell receptor (NKp30) recognition by NK cells by shedding B7-H6 from their cell surface (102). Interestingly, the study observed significantly elevated levels of soluble B7-H6 in the sera of patients with melanoma compared with sera of healthy donors. There is also interest in what prevents NK cells from entering tumors. In mice, a recent study reported the importance of tumor-derived IL-17D in recruiting NK cells into tumors (103). Interestingly, IL-17D gene expression was decreased in metastatic prostate tumors compared with expression levels in primary prostate tumors and was decreased in more advanced gliomas. NK cells are generally recognized to protect mice from tumor metastasis; however, this role is less well established in humans. NK cell control of hematological malignancies is an area of active interest, given that NK cells normally crosstalk with other lymphocytes and myeloid cells.

Cytokines, chemokines, and metabolic factors. A number of other soluble factors also control the composition and function of the cellular effectors and regulators in the tumor microenvironment. Intriguingly, a high density of $\mathrm{CD}^{+} \mathrm{T}$ cells correlated with shorter survival in $\operatorname{RCC}(79,104)$, ocular melanoma (105), and Hodgkin lymphoma (106). Cellular and molecular analyses of the immune microenvironment of CRC and RCC suggest that the difference may be due to strong expression of immunosuppressive cytokines (TGF- $\beta$ and IL-10), inflammatory cytokines (IL-6 and TNF), and angiogenic factors (VEGF) in these cancers (107). Notably, high levels of VEGF were also shown to negate the positive influence of infiltrating Th1/CD $8^{+} \mathrm{T}$ cells in ovarian and colorectal cancer $(108,109)$. In addition, evidence is now emerging that links hypoxia-induced angiogenesis with immune tolerance (110). Altered glucose metabolism (an emerging hallmark of cancer) (2) and acidification have also been linked with denaturation of IFN- $\gamma$ and suppression of TNF production (111). In addition, recent progress in imaging technologies has highlighted an underappreciated role for the extracellular matrix (ECM) in modulating the effector function of T cells (112). A light reticular fiber network, rather than a dense cross-linked matrix, may facilitate $\mathrm{T}$ cell migration and immunosurveillance in the tumor stroma. Finally, recent studies have also uncovered a role for carcinoma-associated fibroblasts (CAFs) expressing fibroblast activation protein (FAP) and escape from immune surveillance through production of CXCL12 $(113,114)$.

The impact of the local education of $\mathrm{T}$ and $\mathrm{B}$ cells in CRC is enforced by the fact that in patients with tumors in which the gene encoding CXCL13 (a TLS-generating chemokine) was deleted, the tumors were less infiltrated by Tfh and B cells, and the patients had a shorter survival (18). IL-15 promotes lymphocyte survival and differentiation, and patients whose tumors had lost IL15 had shorter survival (115). In mice, it was shown that cutaneous tumors ceased CXCL9 production as a result of IFN- $\gamma$-mediated immunoediting (116). These tumors acquired resistance to T cell-mediated immunity and were less able to recruit $\mathrm{T}$ and NK cells. T cell effector function can also be suppressed by inhibitory metabolic enzymes such as indolamine $2{ }^{\prime} 3^{\prime}$-dioxygenase (IDO), an IFN- $\gamma$ induced gene that is significantly upregulated in patients with 
CRC-MSI, (57). Overall, these data illustrate that immune surveillance of human cancers is complex and that many parameters can influence the effector function of $\mathrm{CD}^{+} \mathrm{T}$ cells.

\section{The role of cancer immunoediting in metastases}

Most studies on the immune microenvironment have been conducted in primary human tumors, which are typically accessible by curative surgery. These studies have established that the patient's immune reaction can prevent tumor invasion and partly control the metastatic process. Does it mean that once a metastasis has been established in a distant organ, cancer has escaped immune surveillance? This question can begin to be approached by sampling metastatic sites that undergo surgery for curative or palliative reasons. Such samples are biased, because only operable metastases (likely the optimal cases) are analyzed; however, there are clearly metastases with high or low densities of TLS and high or low densities of T cells and DCs. In the few cases in which the primary tumors were also available, the immune contexture of the metastatic sites globally recapitulated that of the primary tumor (79). Patients with hepatic (117) or lung (79) metastases of $\mathrm{CRC}$ with high $\mathrm{CD} 8^{+} \mathrm{T}$ cell infiltration had a longer survival time than did patients with low $\mathrm{CD} 8^{+} \mathrm{T}$ cell infiltrates, while patients with a high infiltration of $\mathrm{PD}-1^{+} \mathrm{LAG}-3^{+} \mathrm{CD} 8^{+} \mathrm{T}$ cells in RCC lung metastases had a short survival, as was the case with the primary tumor (107). These observations support the concept that the type of immune surveillance in patients is induced early in the evolution of the disease. Operationally, it also explains the success of applying immunotherapeutic approaches in patients with metastatic disease. That the malignant cells are responsible for shaping immune reactions is also supported by the analysis of metastases from different cancer types from the same organ, where the immune contexture reflects the primary tumor and not the organ to which these cells metastasize $(32,79)$.

\section{Conclusion}

Strong evidence now points to the role of cancer immunoediting and $\mathrm{CD}^{+} \mathrm{T}$ cells in sculpting cancer progression, particularly in those tumors displaying an adaptive resistance phenotype. By understanding the immune/tumor microenvironment, we can now target key pathways such as immune checkpoint receptors that suppress endogenous antitumor responses, thereby opening up new avenues of treatment for cancer patients. There are many key questions that must be answered in order for the field to move forward. These include the need to understand the immune contexture of patients with non-type I tumors (nonadaptive immune resistant) so that alternative cancer immunotherapies can be developed for them. In addition, the link between immune cells, vasculature, and other nonimmune elements (e.g., fibroblasts) needs to be better understood. Cancer immunoediting may cause tumor dormancy and/or select and promote cancer cell fitness (stem-like properties). Both MDSCs (118) and Th22 cells (119) have been shown to reshape cancer cells and contribute to cancer progression and metastases. Finally, more information on how cancer immunoediting occurs in epithelial malignancies and the types of tumor microenvironments they display will inform which combinations of immunotherapeutic approaches should translate to the clinic.

Note added in proof. In a recently published article, Le et al. demonstrated that anti-PD-1 induced an objective response rate (ORR) of $40 \%$ in CRC-MSI patients compared with a 0\% ORR in CRC-MSS patients (120).

\section{Acknowledgments}

We apologize to all the authors whose work we were unable to cite because of reference limits. We thank Shin Foong Ngiow for his help drawing Figure 2. M.W.L. Teng is supported by a National Health and Medical Research Council of Australia (NH\&MRC) CDF1 Fellowship grant (1025552), NH\&MRC project grants (1021139 and 1059862), a grant from the Prostate Cancer Foundation of Australia (YI0510), and Cancer Council of Queensland (CCQ) grant (1079876). M.J. Smyth is supported by an NH\&MRC Program Grant (1013667), an NH\&MRC Senior Principal Research Fellowship (1078671), a Susan Komen for the Cure grant (IIR12221504), and a CCQ grant (1083776). W.H. Fridman and J. Galon are members of the ImmunoOncology Laboratory of Excellence and the Cancer Research for Personalized Medicine (CARPEM) program. We thank Catherine Sautès-Fridman for her critical reading of the manuscript and Yann Vano for his help finalizing the manuscript.

Address correspondence to: Mark Smyth, QIMR Berghofer Medical Research Institute, 300 Herston Rd., Herston, UNK 4006, Australia.Phone: 783453957; E-mail:mark.smyth@qimrberghofer.edu.au.
1. Schreiber RD, Old LJ, Smyth MJ. Cancer immunoediting: integrating immunity's roles in cancer suppression and promotion. Science. 2011;331(6024):1565-1570.

2. Hanahan D, Weinberg RA. Hallmarks of cancer: the next generation. Cell. 2011;144(5):646-674.

3. Dunn GP, Old LJ, Schreiber RD. The immunobiology of cancer immunosurveillance and immunoediting. Immunity. 2004;21(2):137-148.

4. Diamond MS, et al. Type I interferon is selectively required by dendritic cells for immune rejection of tumors. JExp Med. 2011;208(10):1989-2003.

5. Vesely MD, Kershaw MH, Schreiber RD, Smyth MJ. Natural innate and adaptive immunity to cancer. Annu Rev Immunol. 2011;29:235-271.

6. Mittal D, Gubin MM, Schreiber RD, Smyth MJ. New insights into cancer immunoediting and its three component phases - elimination, equilibrium and escape. Curr Opin Immunol. 2014;27:16-25.

7. Koebel CM, et al. Adaptive immunity maintains occult cancer in an equilibrium state. Nature. 2007;450(7171):903-907.

8. Teng MW, et al. Opposing roles for IL-23 and IL-12 in maintaining occult cancer in an equilibrium state. Cancer Res. 2012;72(16):3987-3996.

9. Ngiow SF, Teng MW, Smyth MJ. A balance of interleukin-12 and -23 in cancer. Trends Immunol. 2013;34(11):548-555.

10. Muller-Hermelink N, et al. TNFR1 signaling and IFN- $\gamma$ signaling determine whether $\mathrm{T}$ cells induce tumor dormancy or promote multistage carcinogenesis. Cancer Cell. 2008;13(6):507-518.

11. Braumuller H, et al. T-helper-1-cell cyto- kines drive cancer into senescence. Nature. 2013;494(7437):361-365.

12. Matsushita $\mathrm{H}$, et al. Cancer exome analysis reveals a T-cell-dependent mechanism of cancer immunoediting. Nature. 2012;482(7385):400-404.

13. Ferradini L, et al. Analysis of $\mathrm{T}$ cell receptor variability in tumor-infiltrating lymphocytes from a human regressive melanoma. Evidence for in situ T cell clonal expansion. J Clin Invest. 1993;91(3):1183-1190.

14. Zorn E, Hercend T. A natural cytotoxic T cell response in a spontaneously regressing human melanoma targets a neoantigen resulting from a somatic point mutation. Eur J Immunol. 1999;29(2):592-601.

15. Knuth A, Danowski B, Oettgen HF, Old LJ. T-cell-mediated cytotoxicity against autologous 
malignant melanoma: analysis with interleukin 2-dependent T-cell cultures. Proc Natl Acad Sci U S A. 1984;81(11):3511-3515.

16. Fridman WH, Pages F, Sautes-Fridman C, Galon $J$. The immune contexture in human tumours: impact on clinical outcome. Nat Rev Cancer. 2012;12(4):298-306.

17. Page DB, Postow MA, Callahan MK, Allison JP, Wolchok JD. Immune modulation in cancer with antibodies. Annu Rev Med. 2014;65:185-202.

18. Bindea G, et al. Spatiotemporal dynamics of intratumoral immune cells reveal the immune landscape in human cancer. Immunity. 2013;39(4):782-795.

19. Angell H, Galon J. From the immune contexture to the Immunoscore: the role of prognostic and predictive immune markers in cancer. Curr Opin Immunol. 2013;25(2):261-267.

20. Galon J, Fridman WH, Pages F. The adaptive immunologic microenvironment in colorectal cancer: a novel perspective. Cancer Res. 2007;67(5):1883-1886.

21. Pages F, et al. In situ cytotoxic and memory $\mathrm{T}$ cells predict outcome in patients with early-stage colorectal cancer. J Clin Oncol. 2009;27(35):5944-5951.

22. Galon J, et al. Type, density, and location of immune cells within human colorectal tumors predict clinical outcome. Science. 2006;313(5795):1960-1964.

23. Mlecnik B, et al. Histopathologic-based prognostic factors of colorectal cancers are associated with the state of the local immune reaction. J Clin Oncol. 2011;29(6):610-618.

24. Pages F, et al. Effector memory T cells, early metastasis, and survival in colorectal cancer. N Engl J Med. 2005;353(25):2654-2666.

25. Galon J, Angell HK, Bedognetti D, Marincola FM. The continuum of cancer immunosurveillance: prognostic, predictive, and mechanistic signatures. Immunity. 2013;39(1):11-26.

26. Galon J, et al. Cancer classification using the Immunoscore: a worldwide task force. J Transl Med. 2012;10:205.

27. Galon J, et al. Towards the introduction of the 'Immunoscore' in the classification of malignant tumours. J Pathol. 2014;232(2):199-209.

28. Brahmer JR, et al. Safety and activity of antiPD-L1 antibody in patients with advanced cancer. NEngl J Med. 2012;366(26):2455-2465.

29. Hodi FS, et al. Improved survival with ipilimumab in patients with metastatic melanoma. $N$ Engl J Med. 2010;363(8):711-723.

30. Robert C, et al. Ipilimumab plus dacarbazine for previously untreated metastatic melanoma. N Engl J Med. 2011;364(26):2517-2526.

31. Topalian SL, et al. Safety, activity, and immune correlates of anti-PD-1 antibody in cancer. $N$ Engl JMed. 2012;366(26):2443-2454.

32. Dieu-Nosjean MC, Goc J, Giraldo NA, SautesFridman C, Fridman WH. Tertiary lymphoid structures in cancer and beyond. Trends Immunol. 2014;35(11):571-580.

33. Boehm T, Iwanami N, Hess I. Evolution of the immune system in the lower vertebrates. Annu Rev Genomics Hum Genet. 2012;13:127-149.

34. Moyron-Quiroz JE, et al. Role of inducible bronchus associated lymphoid tissue (iBALT) in respi- ratory immunity. Nat Med. 2004;10(9):927-934.

35. Germain C, et al. Presence of B cells in tertiary lymphoid structures is associated with a protective immunity in patients with lung cancer. Am J Respir Crit Care Med. 2014;189(7):832-844.

36. Goc J, Fridman WH, Hammond SA, Sautes-Fridman C, Dieu-Nosjean MC. Tertiary lymphoid structures in human lung cancers, a new driver of antitumor immune responses. Oncoimmunology. 2014;3:e28976

37. Maldonado L, et al. Intramuscular therapeutic vaccination targeting HPV16 induces $\mathrm{T}$ cel responses that localize in mucosal lesions. Sci Transl Med. 2014;6(221):221ra13.

38. Lutz ER, et al. Immunotherapy converts nonimmunogenic pancreatic tumors into immunogenic foci of immune regulation. Cancer Immunol Res. 2014;2(7):616-631.

39. Goc J, et al. Dendritic cells in tumor-associated tertiary lymphoid structures signal a Th1 cytotoxic immune contexture and license the positive prognostic value of infiltrating CD8 ${ }^{+} \mathrm{T}$ cells. Cancer Res. 2014;74(3):705-715.

40. Di Caro G, et al. Occurrence of tertiary lymphoid tissue is associated with $\mathrm{T}$-cell infiltration and predicts better prognosis in early-stage colorectal cancers. Clin Cancer Res. 2014;20(8):2147-2158.

41. Giraldo N, et al. Orchestration and prognostic significance of immune checkpoints in the microenvironment of primary and metastatic renal cell cancer [published online ahead of print February 16, 2015]. Clin Cancer Res. doi:10.1158/10780432.CCR-14-2926.

42. Savas P, Caramia F, Teo ZL, Loi S. Oncogene addiction and immunity: clinical implications of tumour infiltrating lymphocytes in breast cancers overexpressing the HER2/neu oncogene. Curr Opin Oncol. 2014;26(6):562-567.

43. Rabinovich GA, Gabrilovich D, Sotomayor EM. Immunosuppressive strategies that are mediated by tumor cells. Annu Rev Immunol. 2007;25:267-296.

44. Taube JM, et al. Colocalization of inflammatory response with B7-h1 expression in human melanocytic lesions supports an adaptive resistance mechanism of immune escape. Sci Transl Med. 2012;4(127):127ra37.

45. Pardoll DM. The blockade of immune checkpoints in cancer immunotherapy. Nat Rev Cancer. 2012;12(4):252-264.

46. Curiel TJ, et al. Blockade of B7-H1 improves mye loid dendritic cell-mediated antitumor immunity. Nat Med. 2003;9(5):562-567.

47. Kryczek I, et al. B7-H4 expression identifies a novel suppressive macrophage population in human ovarian carcinoma. J Exp Med. 2006;203(4):871-881.

48. Hamid O, et al. Safety and tumor responses with lambrolizumab (anti-PD-1) in melanoma. N Engl JMed. 2013;369(2):134-144.

49. Wolchok JD, Chan TA. Cancer: Antitumour immunity gets a boost. Nature. 2014;515(7528):496-498.

50. Herbst RS, et al. Predictive correlates of response to the anti-PD-L1 antibody MPDL3280A in cancer patients. Nature. 2014;515(7528):563-567.

51. Taube JM, et al. Association of PD-1, PD-1 ligands, and other features of the tumor immune microenvironment with response to anti-PD-1 therapy.
Clin Cancer Res. 2014; 20(19):5064-5074.

52. Tumeh PC, et al. PD-1 blockade induces responses by inhibiting adaptive immune resistance. Nature. 2014;515(7528):568-571.

53. Parsa AT, et al. Loss of tumor suppressor PTEN function increases B7-H1 expression and immunoresistance in glioma. Nat Med. 2007;13(1):84-88

54. Atefi M, et al. Effects of MAPK and PI3K pathways on PD-L1 expression in melanoma. Clin Cancer Res. 2014;20(13):3446-3457.

55. Gros A, et al. PD-1 identifies the patient-specific CD8(+) tumor-reactive repertoire infiltrating human tumors. J Clin Invest. 2014; 124(5):2246-2259.

56. Saeterdal I, et al. Frameshift-mutation-derived peptides as tumor-specific antigens in inherited and spontaneous colorectal cancer. Proc Natl Acad Sci U S A. 2001;98(23):13255-13260.

57. Llosa NJ, et al. The vigorous immune microenvironment of microsatellite instable colon cancer is balanced by multiple counter-inhibitory checkpoints. Cancer Discov. 2015;5(1):43-51.

58. Prall F, et al. Prognostic role of CD8+ tumor-infiltrating lymphocytes in stage III colorectal cancer with and without microsatellite instability. Hum Pathol. 2004;35(7):808-816.

59. Gubin MM, et al. Checkpoint blockade cancer immunotherapy targets tumour-specific mutant antigens. Nature. 2014;515(7528):577-581.

60. Yadav M, et al. Predicting immunogenic tumour mutations by combining mass spectrometry and exome sequencing. Nature. 2014;515(7528):572-576

61. Gerlinger $\mathrm{M}$, et al. Intratumor heterogeneity and branched evolution revealed by multiregion sequencing. NEngl JMed. 2012;366(10):883-892

62. von Boehmer L, et al. NY-ESO-1-specific immunological pressure and escape in a patient with metastatic melanoma. Cancer Immun. 2013;13:12.

63. Sucker A, et al. Genetic evolution of T-cell resistance in the course of melanoma progression. Clin Cancer Res. 2014;20(24):6593-6604.

64. Shankaran V, et al. IFN $\gamma$ and lymphocytes prevent primary tumour development and shape tumour immunogenicity. Nature. 2001;410(6832):1107-1111

65. Vogelstein B, Papadopoulos N, Velculescu VE, Zhou S, Diaz LA Jr, Kinzler KW. Cancer genome landscapes. Science. 2013;339(6127):1546-1558.

66. Rooney MS, Shukla SA, Wu CJ, Getz G, Hacohen N. Molecular and Genetic Properties of tumors associated with local immune cytolytic activity. Cell. 2015;160(1-2):48-61.

67. Tran E, et al. Cancer immunotherapy based on mutation-specific $\mathrm{CD} 4^{+} \mathrm{T}$ cells in a patient with epithelial cancer. Science. 2014;344(6184):641-645.

68. Lavoue V, et al. Immunity of human epithelial ovarian carcinoma: the paradigm of immune suppression in cancer. J Transl Med. 2013;11:147.

69. Ioannides CG, Fisk B, Fan D, Biddison WE, Wharton JT, O'Brian CA. Cytotoxic T cells isolated from ovarian malignant ascites recognize a peptide derived from the HER-2/neu proto-oncogene. Cell Immunol. 1993;151(1):225-234.

70. Curtis C, et al. The genomic and transcriptomic architecture of 2,000 breast tumours reveals novel subgroups. Nature. 2012;486(7403):346-352. 
71. Stagg J, et al. Anti-ErbB-2 mAb therapy requires type I and II interferons and synergizes with antiPD-1 or anti-CD137 mAb therapy. Proc Natl Acad Sci U S A. 2011;108(17):7142-7147.

72. [No authors listed]. Pembrolizumab shows potential in breast cancer. Cancer Discov. 2014; $5(2): 100-101$

73. Gabrilovich DI, Ostrand-Rosenberg S, Bronte V. Coordinated regulation of myeloid cells by tumours. Nat Rev Immunol. 2012;12(4):253-268.

74. Lewis CE, Pollard JW. Distinct role of macrophages in different tumor microenvironments. Cancer Res. 2006;66(2):605-612.

75. Affara NI, et al. B cells regulate macrophage phenotype and response to chemotherapy in squamous carcinomas. Cancer Cell. 2014;25(6):809-821.

76. Ruffell B, Au A, Rugo HS, Esserman LJ, Hwang ES, Coussens LM. Leukocyte composition of human breast cancer. Proc Natl Acad Sci U S A. 2012;109(8):2796-2801.

77. Dieu-Nosjean MC, et al. Long-term survival for patients with non-small-cell lung cancer with intratumoral lymphoid structures. J Clin Oncol. 2008;26(27):4410-4417.

78. Martinet L, et al. Human solid tumors contain high endothelial venules: association with $\mathrm{T}$ - and B-lymphocyte infiltration and favorable prognosis in breast cancer. Cancer Res. 2011;71(17):5678-5687.

79. Remark R, et al. Characteristics and clinical impacts of the immune environments in colorectal and renal cell carcinoma lung metastases: influence of tumor origin. Clin Cancer Res. 2013;19(15):4079-4091.

80. Scarlett UK, et al. Ovarian cancer progression is controlled by phenotypic changes in dendritic cells. JExp Med. 2012;209(3):495-506.

81. Berger-Achituv S, et al. A proposed role for neutrophil extracellular traps in cancer immunoediting. Front Immunol. 2013;4:48.

82. Cools-Lartigue J, et al. Neutrophil extracellular traps sequester circulating tumor cells promote metastasis. J Clin Invest. 2013;123(8):3446-3458.

83. Schreck S, et al. Prognostic impact of tumour-infiltrating Th2 and regulatory T cells in classical Hodgkin lymphoma. Hematol Oncol. 2009;27(1):31-39.

84. Yoon NK, et al. Higher levels of GATA3 predict better survival in women with breast cancer. Hum Pathol. 2010;41(12):1794-1801.

85. Kusuda T, Shigemasa K, Arihiro K, Fujii T, Nagai N, Ohama K. Relative expression levels of Th1 and Th2 cytokine mRNA are independent prognostic factors in patients with ovarian cancer. Oncol Rep. 2005;13(6):1153-1158.

86. De Monte L, et al. Intratumor T helper type 2 cell infiltrate correlates with cancer-associated fibroblast thymic stromal lymphopoietin production and reduced survival in pancreatic cancer. JExp Med. 2011;208(3):469-478.

87. Ubukata H, Motohashi G, Tabuchi T, Nagata H, Konishi S. Evaluations of interferon- $\gamma /$ interleukin-4 ratio and neutrophil/lymphocyte ratio as prognostic indicators in gastric cancer patients. JSurg Oncol. 2010;102(7):742-747.
88. Tosolini M, et al. Clinical impact of different classes of infiltrating T cytotoxic and helper cells (Th1, th2, treg, th17) in patients with colorectal cancer. Cancer Res. 2011;71(4):1263-1271.

89. Chen X, et al. Increased IL-17-producing cells correlate with poor survival and lymphangiogenesis in NSCLC patients. Lung Cancer. 2010;69(3):348-354.

90. Zhang JP, et al. Increased intratumoral IL-17-producing cells correlate with poor survival in hepatocellular carcinoma patients. J Hepatol. 2009;50(5):980-989.

91. Kryczek I, Wei S, Szeliga W, Vatan L, Zou W. Endogenous IL-17 contributes to reduced tumor growth and metastasis. Blood. 2009;114(2):357-359.

92. Lv L, et al. The accumulation and prognosis value of tumor infiltrating IL-17 producing cells in esophageal squamous cell carcinoma. PLoS One. 2011;6(3):e18219.

93. Chen JG, et al. Intratumoral expression of IL-17 and its prognostic role in gastric adenocarcinoma patients. Int J Biol Sci. 2011;7(1):53-60.

94. Curiel TJ, et al. Specific recruitment of regulatory $\mathrm{T}$ cells in ovarian carcinoma fosters immune privilege and predicts reduced survival. Nat Med. 2004;10(9):942-949.

95. Milne K, et al. Systematic analysis of immune infiltrates in high-grade serous ovarian cancer reveals CD20, FoxP3 and TIA-1 as positive prognostic factors. PLoS One. 2009;4(7):e6412.

96. Pastille E, et al. Transient ablation of regulatory $\mathrm{T}$ cells improves antitumor immunity in colitis-associated colon cancer. Cancer Res. 2014;74(16):4258-4269.

97. Tanchot $\mathrm{C}$, et al. Tumor-infiltrating regulatory $\mathrm{T}$ cells: phenotype, role, mechanism of expansion in situ and clinical significance. Cancer Microenviron. 2013;6(2):147-157.

98. Gu-Trantien C, et al. CD4(+) follicular helper T cell infiltration predicts breast cancer survival. J Clin Invest. 2013;123(7):2873-2892.

99. Platonova S, et al. Profound coordinated alterations of intratumoral NK cell phenotype and function in lung carcinoma. Cancer Res. 2011;71(16):5412-5422.

100.Mamessier E, et al. Human breast cancer cells enhance self tolerance by promoting evasion from NK cell antitumor immunity. JClin Invest. 2011;121(9):3609-3622.

101. Tu MM, et al. Ly49 family receptors are required for cancer immunosurveillance mediated by natural killer cells. Cancer Res. 2014;74(14):3684-3694.

102.Schlecker E, et al. Metalloprotease-mediated tumor cell shedding of B7-H6, the ligand of the natural killer cell-activating receptor NKp3O. Cancer Res. 2014;74(13):3429-3440.

103. O'Sullivan T, et al. Interleukin-17D mediates tumor rejection through recruitment of natural killer cells. Cell Rep. 2014;7(4):989-998.

104. Nakano O, et al. Proliferative activity of intratumoral CD8(+) T-lymphocytes as a prognostic fac tor in human renal cell carcinoma: clinicopathologic demonstration of antitumor immunity. Cancer Res. 2001;61(13):5132-5136.
105. Whelchel JC, Farah SE, McLean IW, Burnier MN. Immunohistochemistry of infiltrating lymphocytes in uveal malignant melanoma. Invest Ophthalmol Vis Sci. 1993;34(8):2603-2606.

106.Asano N, et al. Prognostic significance of T-cell or cytotoxic molecules phenotype in classical Hodgkin's lymphoma: a clinicopathologic study. JClin Oncol. 2006;24(28):4626-4633.

107. Giraldo NA, Becht E, Remark R, Damotte D, Sautes-Fridman C, Fridman WH. The immune contexture of primary and metastatic human tumours. Curr Opin Immunol. 2014;27:8-15.

108. Motz GT, Coukos G. The parallel lives of angiogenesis and immunosuppression: cancer and other tales. Nat Rev Immunol. 2011;11(10):702-711.

109. Camus M, et al. Coordination of intratumoral immune reaction and human colorectal cancer recurrence. Cancer Res. 2009;69(6):2685-2693.

110. Barsoum IB, Koti M, Siemens DR, Graham $\mathrm{CH}$. Mechanisms of hypoxia-mediated immune escape in cancer. Cancer Res. 2014;74(24):7185-7190.

111. Kareva I, Hahnfeldt P. The emerging "hallmarks" of metabolic reprogramming and immune evasion: distinct or linked? Cancer Res. 2013;73(9):2737-2742.

112. Peranzoni E, Rivas-Caicedo A, Bougherara H, Salmon H, Donnadieu E. Positive and negative influence of the matrix architecture on antitumor immune surveillance. Cell Mol Life Sci. 2013;70(23):4431-4448.

113. Fearon DT. The carcinoma-associated fibroblast expressing fibroblast activation protein and escape from immune surveillance. Cancer Immunol Res. 2014;2(3):187-193.

114. Feig C, et al. Targeting CXCL12 from FAPexpressing carcinoma-associated fibroblasts synergizes with anti-PD-L1 immunotherapy in pancreatic cancer. Proc Natl Acad Sci U S A. 2013;110(50):20212-20217.

115. Mlecnik B, et al. Functional network pipeline reveals genetic determinants associated with in situ lymphocyte proliferation and survival of cancer patients. Sci Transl Med. 2014;6(228):228ra37.

116. Petro M, et al. Cutaneous tumors cease CXCL9/ Mig production as a result of IFN- $\gamma$-mediated immunoediting. J Immunol. 2013;190(2):832-841.

117. Halama N, et al. Localization and density of immune cells in the invasive margin of human colorectal cancer liver metastases are prognostic for response to chemotherapy. Cancer Res. 2011;71(17):5670-5677.

118. Cui TX, et al. Myeloid-derived suppressor cells enhance stemness of cancer cells by inducing microRNA101 and suppressing the corepressor CtBP2. Immunity. 2013;39(3):611-621.

119. Kryczek I, et al. IL-22(+)CD4(+) T cells promote colorectal cancer stemness via STAT3 transcription factor activation and induction of the methyltransferase DOT1L. Immunity. 2014;40(5):772-784

120. Le DT, et al. PD-1 blockade in tumors with mismatch-repair deficiency. $N$ Engl J Med. 2015;372(26):2509-2520. 